\title{
Patient voice
}

\section{The treatment burden of cystic fibrosis: a day-to-day experience with treatment as someone with cystic fibrosis}

Jessica Maetz, who was born in 1983 and now lives in Paris, talks about her experiences of life with cystic fibrosis (CF) and how treatment is central to many aspects of her life. Jessica has also produced a short film that gives a day in the life view of her treatments (see the supplementary material).

Doing her best to inspire others with CF, Jessica has also written a book "Short of Breath, Full of Life: Stronger than Cystic Fibrosis" (independently published, 2018), and she has started to write a weekly blog, sharing her experience of working, travelling, being a mum, and all other aspects of life as someone with CF.

My name is Jessica and I am 37 years old. Due to digestive complications at birth, I was diagnosed with CF at a very early stage. Nowadays, all newborns are screened for several genetic diseases 2 days after birth with a blood sample, but in 1983, this was a different story.

I was lucky to be diagnosed early and so I started CF treatment very early. When the CF faulty gene was discovered in 1989, I started primary school. I could manage CF treatment and studies, and in 2002, I entered business school. At the end of my Master's degree, I felt as normal as any other student and I accepted a job offer in London, but only then did I realise that working life was not so easily adapted to having CF as school was. After losing 35 points of breathing capacity, my doctor announced that I was too low to get pregnant, and so I decided to leave London. I moved to Madrid, I set a time limit regarding my working hours and I took more time for my daily care, especially as I was diagnosed with CF-related diabetes shortly afterwards.

My daily treatment consists of physiotherapy, physical exercise, rest, and medication. I take bronchodilator drugs, to open the airways of the lungs, every morning and evening, and also just before a physiotherapy session. I take antibiotics every day to control persistent lung infection, both orally and by nebuliser. Also, I need additional vitamins, such as vitamin D, E and calcium. As my pancreas does not produce enzymes, I need to take some capsules to replace pancreatic enzymes

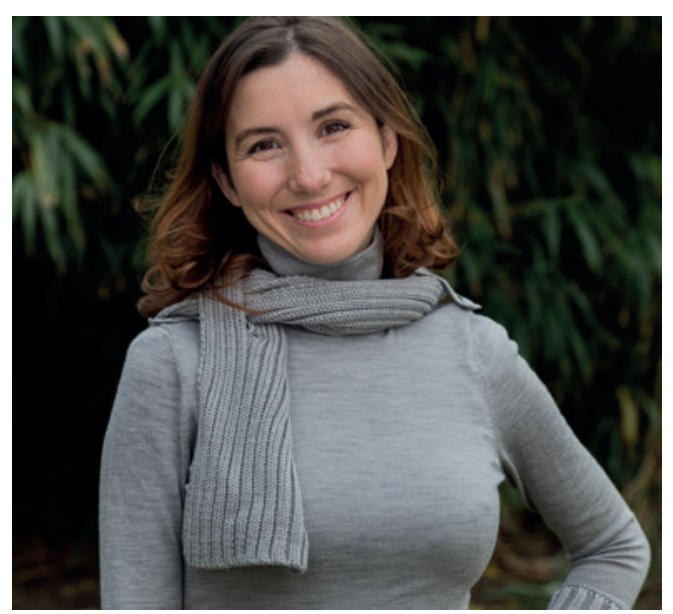

Jessica Maetz.
Cite as: Maetz J, Denning J. The treatment burden of cystic fibrosis: a day-to-day experience with treatment as someone with cystic fibrosis. Breathe 2021; 17: 210013.

@ERSpublications

Treatment for cystic fibrosis interweaves with daily life and can be burdensome, but hope is ever present with the continuing advancement of treatment. https://bit.ly/36PZnSL

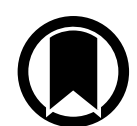

CrossMark 
with any meal, in order to break down food more effectively. It has been a decade since I was diagnosed with CF-related diabetes, so I also need to take insulin with every meal. I have been suffering from ear, nose and throat disorders for 30 years, so I also need to take nasal steroid sprays and wash my nose with salty water to control the evolution of nasal polyps.

To sum up, every day I ingest around 20 tablets, I take six steroid or bronchodilator sprays, I take at least four doses of insulin, and I do a physiotherapy session of airway clearance, that lasts for $30 \mathrm{~min}$.

When I suffer from a lung infection, I receive additional antibiotic treatment, first orally, and if this is not sufficient, intravenously (3-4 times a year). Usually, I also do two airway clearance sessions with my physiotherapist during a lung infection.

Exercise is particularly important for people with CF, so we are encouraged to stay active and to practice daily physical activity. I walk for $1 \mathrm{~h}$ a day and I used to go to ballet once a week (before COVID-19). Since April 2020, I do some physical muscle strengthening exercises at home, always finishing with stretches.

Regarding my CF diet, I need to eat a bit more than other people (I take around 30\% more in quantity than a healthy person) as my body needs extra calories to be able to fight infection. As my pancreas is not functioning correctly, I tend to avoid all fat that is more difficult to digest. I also need additional salt as my body does not retain it, and sufficient hydration. Usually, I drink 2 litres of water a day. Correctly feeding the body can be really challenging when feeling tired or lacking appetite and sometimes oral nutritional supplements are needed to increase caloric intake.

It is hard to sleep well with CF as you often suffer from crisis of cough, especially at night. That is why I sleep with a big pillow to keep me seated. Coughing all the time is exhausting, for your body and for your brain (and for other people living under the same roof as you!).

Regarding other aspects of the disease, that are still considered as taboo by many patients, I will tell you about my experience of urinary incontinence. Women with CF are indeed more likely to experience urinary incontinence than the healthy population, due to chronic and prolonged cough. For many years, I found it impossible to discuss the matter with my doctor, as I felt too ashamed. It is nobler to suffer from a lung infection than from bladder leaks. Then, I met a physiotherapist who was offering sessions to control leakage, teaching how to tighten and lift the pelvic floor muscles. Although I was regularly practising, I could not stop it when coughing and I felt all the more embarrassed. After pregnancy it got even worse. Finally, I decided to speak to my doctor after two episodes of huge leaks, and she sent me to a urologist. After a series of exams with him, he proposed a small operation under local anaesthesia. $24 \mathrm{~h}$ later, the issue was solved for good. I still feel amazed at how simple the solution to my problem was, and if I had been able to talk to my doctor sooner, I would have suffered much less.

The challenges of being an adult with CF are so complex that I needed help. First you have to deal with all the challenges linked to CF itself and then the fact that it often gets worse in early adulthood when it is important to build a social life. That means that you have to manage your daily treatments on your own and maintain going out with your friends and doing your activities. On top of that, for young adults with CF looking for a life-partner can be tricky. We have a tendency to have low self-confidence and it is also hard to project oneself into bigger plans for the future, especially parenting as an adult with CF.

Finally, as we live in a society, we need to fulfil its rules to be integrated. That means doing the same things that all our peers: studying, working, traveling, etc. When you do not follow the rules, it is harder, and not only from a financial point of view. You need additional strength to fight other people's prejudices and that is not easy as fighting CF also requires a lot of energy.

The critical point for an orphan disease is to be able to build a whole community, including patients, therapists, investigators, volunteers, etc. For that aspect, CF is a great example of a successful community. We all have a role to play. As patients, we need to stick to our treatments and to volunteer for clinical research as much as possible.

In the past 35 years, we have achieved many positive things, leading to a better life quality for all CF patients. With new and very promising developments happening in CF research and treatment, I completely trust that the next 35 years will be even better, and that is the reason why I am enjoying life so much!

\section{Affiliations}

Jessica Maetz ${ }^{1,2}$, Jessica Denning ${ }^{1}$

${ }^{1}$ European Lung Foundation, Sheffield, UK. ${ }^{2}$ Vaincre la mucoviscidose, Paris, France.

\section{Supplementary material}

This article has supplementary material available from breathe.ersjournals.com

\section{Conflict of interest}

J. Denning is an employee of the European Lung Foundation. 